\title{
Use of a 2-Dose Schedule for Human Papillomavirus Vaccination - Updated Recommendations of the Advisory Committee on Immunization Practices
}

\author{
Elissa Meites, $\mathrm{MD}^{1}$; Allison Kempe, $\mathrm{MD}^{2,3}$; Lauri E. Markowitz, $\mathrm{MD}^{1}$
}

\section{Introduction}

Vaccination against human papillomavirus (HPV) is recommended to prevent HPV infections and HPV-associated diseases, including cancers. Routine vaccination at age 11 or 12 years has been recommended by the Advisory Committee on Immunization Practices (ACIP) since 2006 for females and since 2011 for males $(1,2)$. This report provides recommendations and guidance regarding use of HPV vaccines and updates ACIP HPV vaccination recommendations previously published in 2014 and 2015 (1,2). This report includes new recommendations for use of a 2-dose schedule for girls and boys who initiate the vaccination series at ages 9 through 14 years. Three doses remain recommended for persons who initiate the vaccination series at ages 15 through 26 years and for immunocompromised persons.

\section{Background}

HPV infection causes cervical, vaginal, and vulvar cancers in women; penile cancers in men; and oropharyngeal and anal cancers as well as genital warts in both men and women (3).

Recommendations for use of vaccines in children, adolescents and adults are developed by the Advisory Committee on Immunization Practices (ACIP). ACIP is chartered as a federal advisory committee to provide expert external advice and guidance to the Director of the Centers for Disease Control and Prevention (CDC) on use of vaccines and related agents for the control of vaccinepreventable diseases in the civilian population of the United States. Recommendations for use of vaccines in children and adolescents are harmonized to the greatest extent possible with recommendations made by the American Academy of Pediatrics (AAP), the American Academy of Family Physicians (AAFP), and the American College of Obstetricians and Gynecologists (ACOG). Recommendations for routine use of vaccines in adults are harmonized with recommendations of $A A F P, A C O G$, and the American College of Physicians (ACP). ACIP recommendations approved by the CDC Director become agency guidelines on the date published in the Morbidity and Mortality Weekly Report (MMWR). Additional information about ACIP is available at https://www.cdc.gov/vaccines/acip.
Three HPV vaccines are licensed for use in the United States. All are noninfectious. Quadrivalent and 9-valent HPV vaccines (4vHPV and 9vHPV, Gardasil and Gardasil 9, Merck and Co, Inc., Whitehouse Station, New Jersey) are licensed for use in females and males aged 9 through 26 years (1). Bivalent HPV vaccine (2vHPV, Cervarix, GlaxoSmithKline, Rixensart, Belgium) is licensed for use in females aged 9 through 25 years (1). As of late 2016, only $9 \mathrm{vHPV}$ is being distributed in the United States. The majority of all HPV-associated cancers are caused by HPV 16 or 18, types targeted by all three vaccines. In addition, 4vHPV targets HPV 6 and 11, types that cause genital warts. 9vHPV protects against these and five additional types: HPV 31, 33, 45, 52, and 58. All three vaccines have been approved for administration in a 3-dose series at intervals of 0,1 or 2 , and 6 months. In October 2016, after considering new clinical trial results (4), the Food and Drug Administration (FDA) also approved 9vHPV for use in a 2-dose series for girls and boys aged 9 through 14 years (5). In October 2016, ACIP recommended a 2-dose schedule for adolescents initiating HPV vaccination in this age range. This report provides recommendations for use of 2-dose and 3-dose schedules for $\mathrm{HPV}$ vaccination.

\section{Methods}

During November 2015-October 2016, the ACIP HPV Vaccines Work Group held monthly telephone conferences to 1) review and evaluate the quality of the evidence assessing immunogenicity, efficacy, and postlicensure effectiveness of a 2-dose schedule; 2) consider benefits and harms of a 2-dose schedule; 3 ) weigh the variability in the values and preferences of patients and providers for a 2-dose schedule; and 4) examine health economic analyses. During teleconferences, summaries of findings were presented for Work Group discussion.

A systematic review was conducted to identify studies involving human subjects* that reported primary data on any important or critical health outcomes related to HPV vaccination $^{\dagger}$ after 2 doses of $9 \mathrm{vHPV}, 4 \mathrm{vHPV}$, or $2 \mathrm{vHPV}$, administered at an interval of 0 and $\geq 6$ months ( \pm 4 weeks) to

\footnotetext{
*No primary data on special populations or medical conditions, including immunocompromising conditions, were available for 2-dose intervals and age ranges specified.

$\dagger$ No primary data on other important and critical outcomes, including genital warts, precancers, oropharyngeal cancer, anal cancer, cervical cancer, vaginal/vulvar cancer, and penile cancer, were available for 2-dose intervals and age ranges specified.
} 
persons aged 9 through 14 years. The review focused on this age group given available 2-dose trial data for 9vHPV (4). Immunogenicity outcomes of interest were seroconversion, geometric mean titers (GMTs), or antibody avidity. Studies were excluded if they lacked a comparison group in which efficacy of 3 doses of HPV vaccine against clinical endpoints was demonstrated in clinical trials (e.g., females aged 15 through 26 years). ${ }^{\S}$ Evidence regarding a 3 -dose schedule for HPV vaccine was reviewed previously $(1,2)$.

Quality of evidence was evaluated using the Grading of Recommendations Assessment, Development and Evaluation (GRADE) approach. Detailed methods and GRADE tables can be found online (G). Other studies from the search and from the broader literature informed additional expert guidance that extended beyond the research question addressed formally via GRADE analysis ( 7 ). Evidence was reviewed by the Work Group, summarized, and publicly presented at the February and June 2016 ACIP meetings. CDC vaccine recommendations are developed using the GRADE framework (8). Proposed recommendations were presented, and after a public comment period, were approved unanimously by the voting ACIP members at the October 2016 ACIP meeting.

\section{Summary of Key Findings}

Immunogenicity. In the 9vHPV clinical trial that was the basis for FDA approval of a 2-dose series, participants were girls and boys aged 9 through 14 years, compared with young females aged 16 through 26 years (4). Among 1,377 participants, $\geq 97.9 \%$ seroconverted to all nine vaccine-preventable HPV types by 4 weeks after the last dose. For girls and boys who received 2 doses of 9vHPV 6 months apart (0, 6 month schedule) or 12 months apart (0,12 month schedule), noninferiority criteria were met for seroconversion and GMTs. Furthermore, GMTs were significantly higher for all 9vHPV types among persons aged 9 through 14 years who received 2 doses compared with females aged 16-26 years who received 3 doses (0, 2, 6 month schedule). Six additional studies found similar results for $4 \mathrm{vHPV}$ and $2 \mathrm{vHPV}$ (6). Immunogenicity was found to be noninferior with 2 doses in persons aged 9 through 14 years compared with 3 doses in a group in which clinical efficacy was demonstrated (GRADE evidence type 3).

Efficacy and effectiveness. Although efficacy and postlicensure effectiveness studies were reviewed, none met the inclusion criteria detailed above. The prelicensure HPV vaccine efficacy trials were conducted with 3-dose series; post hoc analyses conducted with data from some of these trials found high efficacy against infection among vaccinees who received 2 doses and

\footnotetext{
\$Studies were excluded when 2-dose interval was not $\geq 5$ months.

Twelve votes to none, with one recusal.
}

those who received 3 doses $(9,10)$. A large study comparing 2 doses with 3 doses also suggested similar efficacy against infection (11). Postlicensure effectiveness studies have found lower effectiveness against various HPV-associated outcomes among vaccinees who received 2 doses compared with those who received 3 doses, but methodologic challenges with these studies limit interpretation of the findings.**

Duration of protection. Through 10 years of follow-up from clinical trials, no evidence of waning protection after a 3-dose series of HPV vaccine has been found (1). Because antibody kinetics are similar with 2-dose and 3-dose series, duration of protection is also expected to be long-lasting after a 2-dose series $(12,13)$.

Health impact and cost-effectiveness modeling. Population-level effectiveness and cost-effectiveness of 2-dose and 3-dose schedules of 9vHPV in the United States have been modeled (14). Assuming both efficacy and duration of protection are similar with either schedule, a 2-dose series would be cost-saving and have similar population impact to a 3-dose series. Even if duration of protection is 20 years for a 2 -dose series and lifelong for a 3-dose series, additional benefits of a 3-dose series would be relatively small, and a 2-dose series would be more cost-effective (14).

\section{Rationale}

HPV vaccines are highly effective and safe, and a powerful prevention tool for reducing HPV infections and HPV-associated cancers $(1,2)$. Based on the available immunogenicity evidence, a 2-dose schedule (0, 6-12 months) will have efficacy equivalent to a 3 -dose schedule $(0,1-2,6$ months) if the HPV vaccination series is initiated before the 15 th birthday (GRADE evidence type 3) (6). ACIP recommends a 2-dose schedule for HPV vaccination of girls and boys who initiate the vaccination series at ages 9 through 14 years (Category A recommendation).

\section{Recommendations}

Routine and catch-up age groups. ACIP recommends routine HPV vaccination at age 11 or 12 years. Vaccination can be given starting at age 9 years. ACIP also recommends vaccination for females through age 26 years and for males through age 21 years who were not adequately vaccinated previously. Males aged 22 through 26 years may be vaccinated. (See also: Special populations, Medical conditions)

Dosing schedules. For persons initiating vaccination before their 15 th birthday, the recommended immunization schedule is 2 doses of HPV vaccine. The second dose should be

\footnotetext{
** In studies conducted in the setting of a 3-dose HPV vaccine recommendation or policy, many 2-dose recipients received HPV vaccine doses at a 1-2 month interval; in addition, 2-dose recipients differed from 3-dose recipients in ways that suggested differences in HPV exposure.
} 
TABLE. Recommended number of doses and intervals for human papillomavirus (HPV) vaccine, by age at series initiation and medical conditions United States, 2016

\begin{tabular}{lcc}
\hline Population & $\begin{array}{c}\text { Recommended number of } \\
\text { HPV vaccine doses }\end{array}$ & $\begin{array}{c}\text { Recommended interval } \\
\text { between doses }\end{array}$ \\
\hline $\begin{array}{l}\text { Persons initiating HPV vaccination at ages 9 through 14 years, }{ }^{*} \text { except } \\
\text { immunocompromised persons }{ }^{\dagger}\end{array}$ & 2 & $0,6-12$ months \\
$\begin{array}{l}\text { Persons initiating HPV vaccination at ages } 15 \text { through } 26 \text { years } \\
\text { immunocompromised persons }{ }^{\dagger} \text { initiating HPV vaccination at ages } 9 \text { through } 26 \text { years }\end{array}$ & 3 & $0,1-2,6$ months ${ }^{* *}$ \\
\hline
\end{tabular}

* ACIP recommends routine HPV vaccination for adolescents at age 11 or 12 years; vaccination may be given starting at age 9 years.

† Persons with primary or secondary immunocompromising conditions that might reduce cell-mediated or humoral immunity (see also: Medical conditions)

$\S$ In a 2-dose schedule of HPV vaccine, the minimum interval between the first and second doses is 5 months.

" For persons who were not adequately vaccinated previously, ACIP recommends vaccination for females through age 26 years and for males through age 21 years; males ages 22 through 26 years may be vaccinated. Vaccination is recommended for some persons aged 22 through 26 years; see Medical conditions and Special populations.

** In a 3-dose schedule of HPV vaccine, the minimum intervals are 4 weeks between the first and second doses, 12 weeks between the second and third doses, and 5 months between the first and third doses.

administered 6-12 months after the first dose (0, 6-12 month schedule) $)^{\dagger \dagger}$ (Table).

For persons initiating vaccination on or after their 15 th birthday, the recommended immunization schedule is 3 doses of HPV vaccine. The second dose should be administered 1-2 months after the first dose, and the third dose should be administered 6 months after the first dose $(0,1-2,6$ month schedule) ${ }^{\S}$ (Table).

Persons vaccinated previously. Persons who initiated vaccination with 9vHPV, 4vHPV, or $2 \mathrm{vHPV}$ before their 15 th birthday, and received 2 doses of any HPV vaccine at the recommended dosing schedule (0, 6-12 months), or 3 doses of any HPV vaccine at the recommended dosing schedule $(0$, $1-2,6$ months), are considered adequately vaccinated.

Persons who initiated vaccination with 9vHPV, 4vHPV, or $2 \mathrm{vHPV}$ on or after their 15 th birthday, and received 3 doses of any HPV vaccine at the recommended dosing schedule, are considered adequately vaccinated.

9vHPV may be used to continue or complete a vaccination series started with 4vHPV or 2vHPV.

For persons who have been adequately vaccinated with $2 \mathrm{vHPV}$ or $4 \mathrm{vHPV}$, there is no ACIP recommendation regarding additional vaccination with 9vHPV.

Interrupted schedules. If the vaccination schedule is interrupted, the series does not need to be restarted. The number of recommended doses is based on age at administration of the first dose.

\footnotetext{
†† In a 2-dose schedule of HPV vaccine, the minimum interval between the first and second doses is 5 months. If the second dose is administered after a shorter interval, a third dose should be administered a minimum of 12 weeks after the second dose and a minimum of 5 months after the first dose.

$\$ \$$ In a 3-dose schedule of HPV vaccine, the minimum intervals are 4 weeks between the first and second doses, 12 weeks between the second and third doses, and 5 months between the first and third doses. If a vaccine dose is administered after a shorter interval, it should be readministered after another minimum interval has elapsed since the most recent dose.
}

Special populations. For children with a history of sexual abuse or assault, ACIP recommends routine HPV vaccination beginning at age 9 years.

For men who have sex with men, 99 ACIP recommends routine $\mathrm{HPV}$ vaccination as for all males, and vaccination through age 26 years for those who were not adequately vaccinated previously.

For transgender persons, ACIP recommends routine HPV vaccination as for all adolescents, and vaccination through age 26 years for those who were not adequately vaccinated previously.

Medical conditions. ACIP recommends vaccination with 3 doses of HPV vaccine (0,1-2, 6 months) for females and males aged 9 through 26 years with primary or secondary immunocompromising conditions that might reduce cell-mediated or humoral immunity, ${ }^{* * *}$ such as B lymphocyte antibody deficiencies, T lymphocyte complete or partial defects, HIV infection, malignant neoplasms, transplantation, autoimmune disease, or immunosuppressive therapy, because immune response to vaccination might be attenuated (Table) ( 7 ).

Contraindications and precautions. Contraindications and precautions, including those related to pregnancy, are unchanged from previous recommendations $(1,2)$. Adverse events occurring after administration of any vaccine should be reported to the Vaccine Adverse Event Reporting System (VAERS). Reports can be submitted to VAERS online, by fax, or by mail. Additional information about VAERS is available by telephone (1-800-822-7967) or online (https://vaers.hhs.gov).

\footnotetext{
99 Including men who identify as gay or bisexual, or who intend to have sex with men.

*** The recommendation for a 3-dose schedule of HPV vaccine does not apply to children aged $<15$ years with asplenia, asthma, chronic granulomatous disease, chronic liver disease, chronic lung disease, chronic renal disease, central nervous system anatomic barrier defects (e.g., cochlear implant), complement deficiency, diabetes, heart disease, or sickle cell disease.
} 


\section{Acknowledgments}

Members of the Advisory Committee on Immunization Practices (ACIP) (member roster for July 2016-June 2017 is available online at https://www.cdc.gov/vaccines/acip/committee/members-archive. html); ACIP HPV Vaccines Work Group: Jorge E. Arana, MD, Atlanta, Georgia; Joseph Bocchini, MD, Shreveport, Louisiana; Harrell Chesson, PhD, Atlanta, Georgia; Tamera Coyne-Beasley, MD, Chapel Hill, North Carolina; C. Robinette Curtis, MD, Atlanta, Georgia; Carolyn D. Deal, PhD, Bethesda, Maryland; Shelley Deeks, MD, Toronto, Ontario, Canada; John Douglas, MD, Greenwood Village, Colorado; Linda Eckert, MD, Seattle, Washington; Sandra Adamson Fryhofer, MD, Atlanta, Georgia; Julianne Gee, MPH, Atlanta, Georgia; Bruce G. Gellin, MD, Washington, DC; Samuel Katz, MD, Durham, North Carolina; Alison Kempe, MD, Denver, Colorado (Chair); Aimée R. Kreimer, PhD, Bethesda, Maryland; Joohee Lee, MD, Silver Spring, Maryland; Lauri E. Markowitz, MD, Atlanta, Georgia (CDC Lead); Elissa Meites, MD, Atlanta, Georgia; Amy B. Middleman, MD, Oklahoma City, Oklahoma; Chris Nyquist, MD, Denver, Colorado; Sean O’Leary, MD, Aurora, Colorado; Sara E. Oliver, MD, Atlanta, Georgia; Cynthia Pellegrini, Washington, DC; Jeff Roberts, MD; Rockville, Maryland; José R. Romero, MD, Little Rock, Arkansas; Jeanne Santoli, MD, Atlanta, Georgia; Mona Saraiya, MD, Atlanta, Georgia; Debbie Saslow, PhD, Atlanta, Georgia; Margot Savoy, MD, Wilmington, Delaware; Shannon Stokley, DrPH, Atlanta, Georgia; Lakshmi Sukumaran, MD, Atlanta, Georgia; Elizabeth R. Unger, PhD, MD, Atlanta, Georgia; Patricia Whitley-Williams, MD, New Brunswick, New Jersey; Rodney Willoughby, MD, Wauwatosa, Wisconsin; JoEllen Wolicki, Atlanta, Georgia; Sixun Yang, MD, Rockville, Maryland; Jane Zucker, MD, New York, New York.

\footnotetext{
${ }^{1}$ Division of Viral Diseases, National Center for Immunization and Respiratory Diseases, CDC; ${ }^{2} \mathrm{HPV}$ Vaccines Work Group, Advisory Committee on Immunization Practices, Atlanta, Georgia; ${ }^{3}$ Department of Pediatrics, University of Colorado Anschutz Medical Campus, Denver, Colorado.
}

Corresponding author: Elissa Meites, emeites@cdc.gov, 404-639-8253.

\section{References}

1. Markowitz LE, Dunne EF, Saraiya M, et al. Human papillomavirus vaccination: recommendations of the Advisory Committee on Immunization Practices (ACIP). MMWR Recomm Rep 2014;63(No. RR-05).

2. Petrosky E, Bocchini JA Jr, Hariri S, et al. Use of 9-valent human papillomavirus (HPV) vaccine: updated HPV vaccination recommendations of the advisory committee on immunization practices. MMWR Morb Mortal Wkly Rep 2015;64:300-4.

3. Viens LJ, Henley SJ, Watson M, et al. Human papillomavirus-associated cancers-United States, 2008-2012. MMWR Morb Mortal Wkly Rep 2016;65:661-6. http://dx.doi.org/10.15585/mmwr.mm6526a1
4. Iversen O-E, Miranda MJ, Ulied A, et al. Immunogenicity of the 9-valent $\mathrm{HPV}$ vaccine using 2-dose regimens in girls and boys vs a 3-dose regimen in women. JAMA 2016;316:2411-21. http://dx.doi.org/10.1001/ jama.2016.17615

5. Food and Drug Administration. Prescribing information [package insert]. Gardasil 9 [human papillomavirus 9-valent vaccine, recombinant]. Silver Spring, MD: US Department of Health and Human Services, Food and Drug Administration; 2016. http://www.fda.gov/downloads/ BiologicsBloodVaccines/Vaccines/ApprovedProducts/UCM426457.pdf

6. CDC. Grading of Recommendations Assessment, Development and Evaluation (GRADE) of a 2-dose schedule for human papillomavirus (HPV) vaccination. Atlanta, GA: US Department of Health and Human Services, CDC; 2016. https://www.cdc.gov/vaccines/acip/recs/grade/ hpv-2-dose.html

7. Rubin LG, Levin MJ, Ljungman P, et al.; Infectious Diseases Society of America. 2013 IDSA clinical practice guideline for vaccination of the immunocompromised host. Clin Infect Dis 2014;58:e44-100. http:// dx.doi.org/10.1093/cid/cit684

8. Ahmed F, Temte JL, Campos-Outcalt D, Schünemann HJ; ACIP Evidence Based Recommendations Work Group (EBRWG). Methods for developing evidence-based recommendations by the Advisory Committee on Immunization Practices (ACIP) of the U.S. Centers for Disease Control and Prevention (CDC). Vaccine 2011;29:9171-6. http://dx.doi.org/10.1016/j.vaccine.2011.08.005

9. Kreimer AR, Struyf F, Del Rosario-Raymundo MR, et al.; Costa Rica Vaccine Trial Study Group Authors; PATRICIA Study Group Authors; HPV PATRICIA Principal Investigators/Co-Principal Investigator Collaborators; GSK Vaccines Clinical Study Support Group. Efficacy of fewer than three doses of an HPV-16/18 AS04-adjuvanted vaccine: combined analysis of data from the Costa Rica Vaccine and PATRICIA Trials. Lancet Oncol 2015;16:775-86. http://dx.doi.org/10.1016/ S1470-2045(15)00047-9

10. Kreimer AR, Rodriguez AC, Hildesheim A, et al.; CVT Vaccine Group. Proof-of-principle evaluation of the efficacy of fewer than three doses of a bivalent HPV16/18 vaccine. J Natl Cancer Inst 2011;103:1444-51. http://dx.doi.org/10.1093/jnci/djr319

11. Sankaranarayanan R, Prabhu PR, Pawlita M, et al.; Indian HPV Vaccine Study Group. Immunogenicity and HPV infection after one, two, and three doses of quadrivalent HPV vaccine in girls in India: a multicentre prospective cohort study. Lancet Oncol 2016;17:67-77. http://dx.doi. org/10.1016/S1470-2045(15)00414-3

12. Romanowski B, Schwarz TF, Ferguson L, et al. Sustained immunogenicity of the HPV-16/18 AS04-adjuvanted vaccine administered as a two-dose schedule in adolescent girls: five-year clinical data and modeling predictions from a randomized study. Hum Vaccin Immunother 2016;12:20-9. http://dx.doi.org/10.1080/21645515.2015.1065363

13. Dobson SR, McNeil S, Dionne M, et al. Immunogenicity of 2 doses of $\mathrm{HPV}$ vaccine in younger adolescents vs 3 doses in young women: a randomized clinical trial. JAMA 2013;309:1793-802. http://dx.doi. org/10.1001/jama.2013.1625

14. Laprise JF, Markowitz LE, Chesson HW, Drolet M, Brisson M. Comparison of 2-dose and 3-dose 9-valent human papillomavirus vaccine schedules in the United States: a cost-effectiveness analysis. J Infect Dis 2016;214:685-8. http://dx.doi.org/10.1093/infdis/jiw227 